\title{
Effect of Ion-Pair Formation on the Rate of Aromatic Substitution
}

\author{
PER BERONIUS
}

\author{
Division of Physical Chemistry, University of Umea, S.901 87 Umeà, Sweden
}

\begin{abstract}
Second-order rate constants for the isotopic exchange between aromatic iodides and potassium iodide in acetone show a marked in. crease with decreasing concentration of the ionic iodide as pointed out by other investigators. This effect was assumed to be caused by variations in the degree of solvation of reactants and transition-state complex with the concentrations of the reactants. In the actual solvent, however, potassium iodide is only partially dissociated and the kinetic salt effect observed might, at least in part, be explained by a much lower reactivity of potassium iodide ion-pairs compared with free iodide ions as discussed in the present article.
\end{abstract}

T he mechanism of substitution of aromatically bound iodine has recently been subjected to investigations by Broadbank, Harhash, and Kanchanalai ${ }^{1}$ and by Marcopoulos ${ }^{2,3}$ who studied the exchange of radioiodine between 1-iodo-2,4-dinitrobenzene and potassium iodide ${ }^{1,3}$ and between 1-iodo-2,4dinitronaphthalene and potassium iodide ${ }^{2,3}$ in acetone.

Rate measurements were made over a broad concentration range of ionic iodide. Second-order rate constants, evaluated using the McKay equation, ${ }^{4}$ show a significant increase with decreasing potassium iodide concentration. Broadbank et al., ${ }^{1}$ assuming this salt to be completely dissociated in acetone, which is incorrect, ascribe this effect to different degrees of solvation of reactants and transition-state complex at different reactant concentrations. According to conductivity data, ${ }^{5}$ potassium iodide is a weak electrolyte in acetone with an ion-pair dissociation constant of $5.57 \times 10^{-3}$.

Also in exchange reactions between aliphatic halides and alkali halides in solvents of not too high dielectric constants, where the alkali halide is only partially dissociated (ion-pair formation), an increase in the second-order rate constant with decreasing salt concentration has been observed; see Ref. 6 for a review on this subject. This effect is due to a much lower reactivity of the alkali halide ion-pairs compared with free halide ions. In fact, it has not been possible to prove that the ion-pairs are involved at all in the exchange reaction. In the opinion of the present author, it appears most reasonable to assume that ion-pair formation is, at least in part, responsible for the kinetic salt ef- 
fect observed in the case of aromatic substitution reactions. Possibly, neglect of this effect is the main reason for the difficulties in interpreting kinetic data for several reactions of this kind.

The purpose of this article is to present a treatment of kinetic data for the above mentioned two aromatic iodine exchange reactions in which the assumed effect of ion-pair formation on the reaction rate has been taken into consideration.

\section{REACTION MECHANISMS}

The two exchange reactions considered may be represented by the gross formula,

$$
\mathrm{AI}+\mathrm{K}^{131} \mathrm{I} \rightleftharpoons \mathrm{A}^{131} \mathrm{I}+\mathrm{KI}
$$

where AI denotes the aromatic iodide, viz. 1-iodo-2,4-dinitrobenzene or 1iodo-2,4-dinitronaphthalene. The concentrations of the organic and the inorganic iodide and the degree of dissociation of the latter are denoted $a, c$, and $\alpha$, respectively.

Assuming that the exchange may take place by $\mathrm{S}_{\mathrm{N}} \mathrm{l}$ as well as by $\mathrm{S}_{\mathrm{N}} 2$ mechanisms, the $S_{N} l$ exchange is independent of the concentration of the ionic reactant, whereas the $\mathrm{S}_{\mathrm{N}} 2$ exchange may be dependent on the concentrations of both free iodide ions and potassium iodide ion-pairs. As previously mentioned it has not been possible to establish any reactivity of alkali halide ion-pairs in exchange reactions involving aliphatic halides and in the following we shall assume the potassium iodide ion-pairs to be kinetically inactive. For the reaction between the organohalide and free iodide ions we shall assume that any dependence of the rate constant on the ionic strength, $I$, may be neglected. This appears to be a plausible assumption for the rather dilute reaction mixtures $(I<0.017)$ used in the studies of Broadbank et al. ${ }^{1}$ and by Marcopoulos. ${ }^{2,3}$ Compare the independence of the second-order rate constant on the ionic strength for the exchange of ${ }^{131} \mathrm{I}$ between ethyl iodide and sodium iodide in methanol, ${ }^{7}$ where this salt is practically completely dissociated, up to a sodium iodide concentration of at least $0.01 \mathrm{M}$ and also when ammonium perchlorate was added to a concentration of $0.059 \mathrm{M}$.

With these assumptions, the rate of exchange, $R$, may be expressed by the equation,

$$
R=k_{1} a+k_{\mathrm{i}} a c \alpha
$$

where $k_{1}$ is a first-order rate constant and $k_{\mathrm{i}}$ a second-order rate constant for reaction between the organic iodide and free iodide ions. This equation may be rewritten in the form,

$$
R / a=k_{1}+k_{\mathrm{i}} c \alpha
$$

which may be employed to evaluate $k_{1}$ and $k_{\mathrm{i}}$ from the intercept and slope, respectively, of the straight line, which should be obtained upon plotting $R / a$ vs. $c \alpha$. 


\section{DISSOCIATION OF ION-PAIRS}

To apply eqn. (2) to kinetic data, it is necessary to know the degree of dissociation, $\alpha$, of the ionic iodide. This quantity was evaluated for all temperatures within the actual range $20-55^{\circ} \mathrm{C}$ from the $25^{\circ} \mathrm{C}$ ion-pair dissociation constant, $5.57 \times 10^{-3}$, because of the lack of precise conductivity data for potassium iodide over the entire temperature range. The Debye-Hückel second approximation was used to calculate activity coefficients. The value $3.5 \AA$, equal to the sum of the crystallographic radii for $\mathrm{K}^{+}$and $\mathrm{I}^{-}$according to Pauling, ${ }^{8}$ was used for the distance of closest approach of the ions in the potassium iodide ion-pair. Dielectric constants for acetone at the different reaction temperatures were calculated from data given in standard tables. ${ }^{9}$

\section{DISCUSSION OF KINETIC DATA}

The dependence of the rate constant ${ }^{2,3}$ on the concentration of potassium iodide for the halogen exchange between this constituent and 1-iodo-2,4dinitronaphthalene is shown in Fig. 1 for various temperatures. The rate constant, $k$, in this graph is defined by the expression, $k=R / a c$.

An alternative representation of these kinetic data is shown in Fig. 2, where $R / a$ has been plotted $v s$. $c \alpha$ according to eqn. (2). The straight lines have been fitted to the experimental points in this figure using a relative deviation least squares method 10 to avoid different weights to points at high and low values of $R / a$. The highest concentration point at $45^{\circ} \mathrm{C}$ and $55^{\circ} \mathrm{C}$, respectively, falls outside the graph (Fig. 2) but has been included in the least squares treatment. For all reaction temperatures, the straight lines pass within ex-

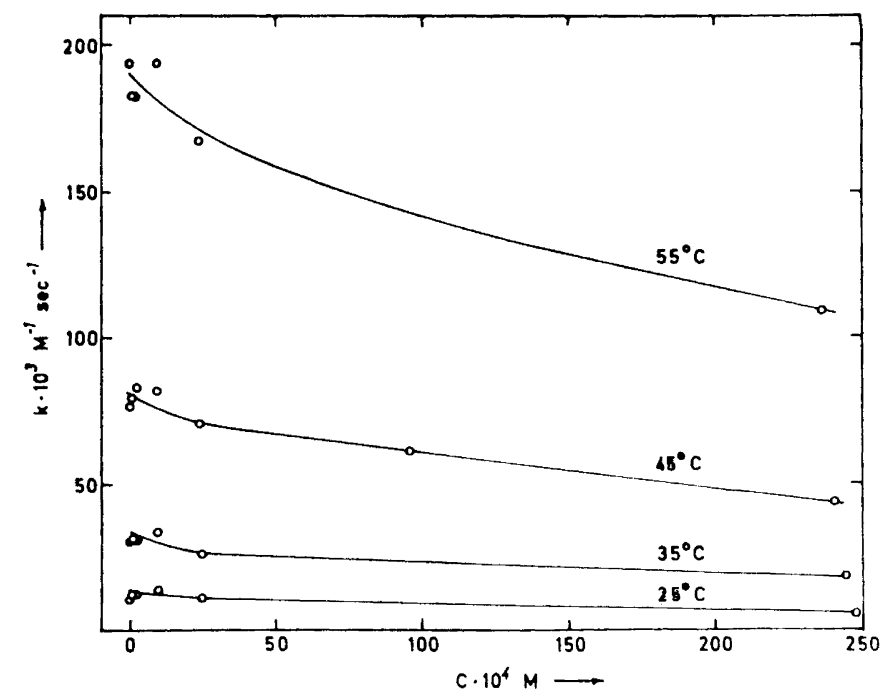

Fig. 1. Dependence of the rate constant, $k$, defined in the text, on the potassium iodide concentration for exchange of ${ }^{131} I$ between this salt and 1-iodo-2,4-dinitronaphthalene in acetone at various temperatures according to Marcopoulos., ${ }^{2,3}$ 
perimental errors through the origin $\left(k_{1}=0\right)$. Hence, there is no evidence for any $\mathrm{S}_{\mathrm{N}}$ 1-contribution to the reaction rate for this system, which appears to be purely second-order.

Fig. 2. Plot of $R / a$ vs. $c \alpha$ for the same system as in Fig. 1 .
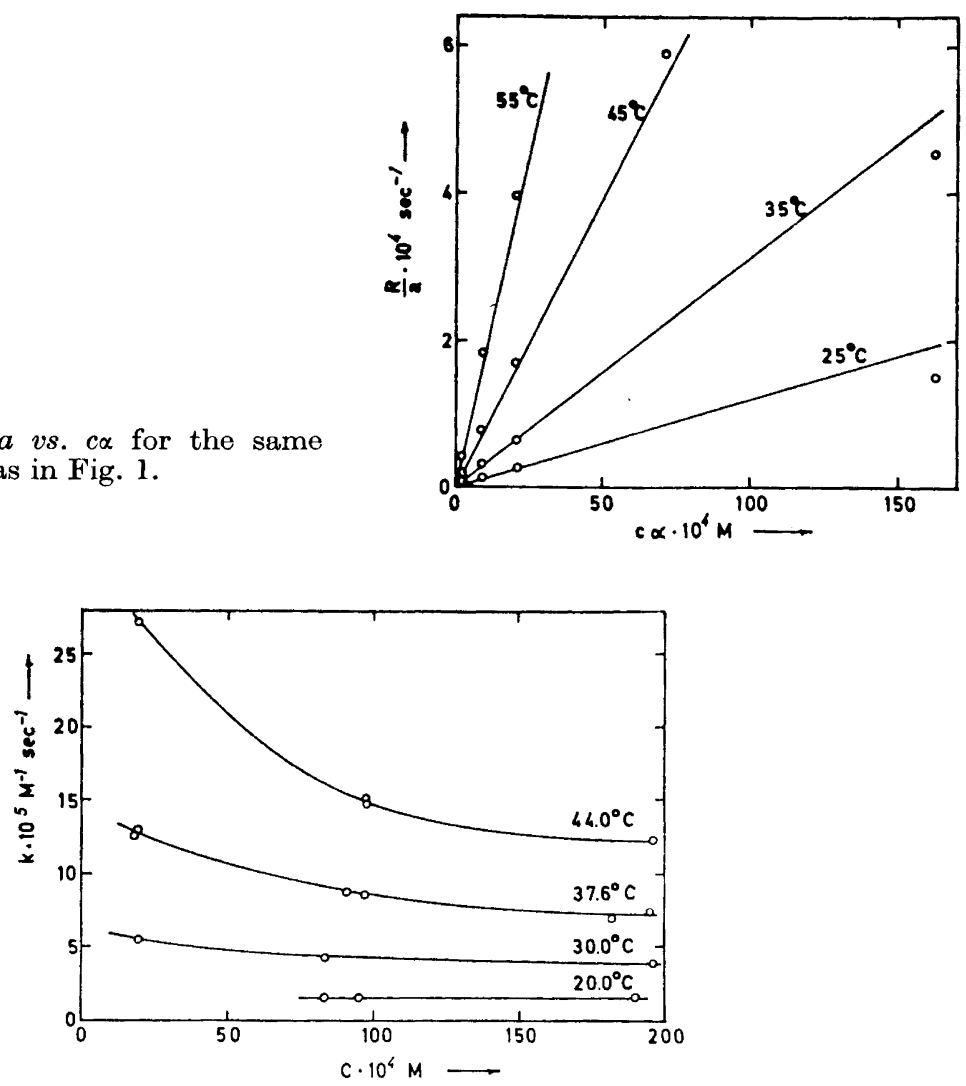

Fig. 3. Dependence of the rate constant, $k$, defined in the text, on the potassium iodide concentration for exchange of ${ }^{131}$ I between this salt and 1-iodo-2,4-dinitrobenzene in acetone at various temperatures according to Broadbank et al. ${ }^{1}$

The corresponding graphs for the isotopic exchange between potassium iodide and 1-iodo-2,4-dinitrobenzene ${ }^{1}$ are given in Figs. 3-4. The intercepts of the lines of $R / a$ vs. $c \alpha$ in Fig. 4, especially at elevated temperatures, indicate that the reaction might be partly of $S_{N} 1$-type. This conclusion must, however, be given with reservation as values of $\alpha$ have been calculated from approximate ion-pair dissociation constants.

Marcopoulos, ${ }^{3}$ referring to a number of articles by various authors concerning the kinetics of isotopic exchange at aromatic carbon atom, makes the comment: "Though kinetic data for aromatic nucleophilic substitutions in various solvents by the use of iodine-131 as radioactive indicator have been published results, as far as solvent effect is concerned, are rather inexplicable". 


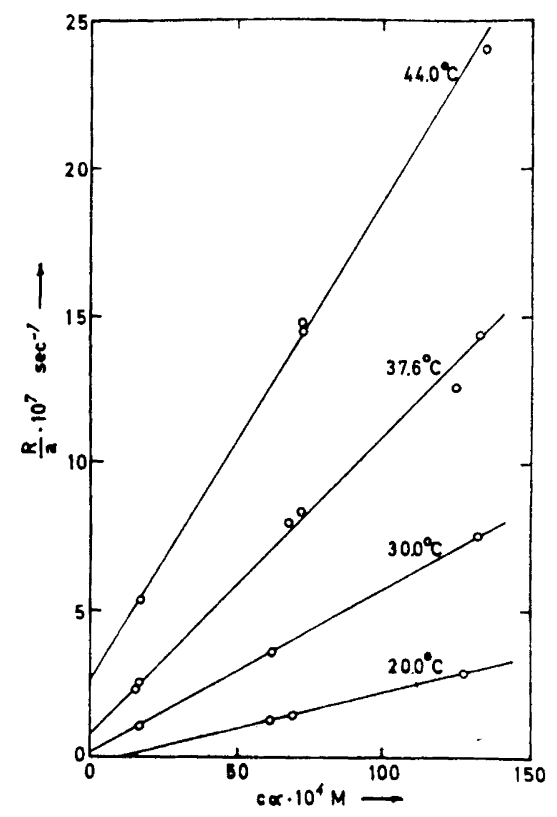

Fig. 4. Plot of $R / a$ vs. $c \alpha$ for the same system as in Fig. 3.

However, the results of the present treatment of kinetic data for aromatic exchange reactions, in which the assumed effect of ion-pair formation has been considered, do not indicate any extraordinary behaviour of this reaction type as compared with aliphatic substitution reactions. The rate data ${ }^{1-3}$ used are, however, not of sufficient precision to permit conclusions as to additional salt effects, e.g. of the type suggested by Broadbank et al. ${ }^{1}$

To obtain more reliable conclusions concerning the role of ion-pair formation and possible other salt effects in aromatic substitution reactions, further experimental studies in this field will be undertaken.

Acknowledgements. The author thanks Dr. Michael Sharp for linguistic revision of the manuscript, and the Swedish Natural Science Research Council for financial support.

\section{REFERENCES}

1. Broadbank, R. W. C., Harhash, A. H. E. and Kanchanalai, S. Radioisotopes Phys. Sci. Ind., Proc. Conf. Use, Copenhagen 1960, 3 (1962) 179.

2. Marcopoulos, C. A. Radiochim. Acta 5 (1966) 121.

3. Marcopoulos, C. A. Z. physik. Chem. (Leipzig) 234 (1967) 297.

4. McKay, H. A. C. J. Am. Chem. Soc. 65 (1943) 702.

5. Savedoff, L. G. J. Am. Chem. Soc. 88 (1966) 664.

6. Beronius, P. Acta Chem. Scand. 23 (1969) 1175.

7. Evans, C. C. and Sugden, S. J. Chem. Soc. 1949270.

8. Pauling, L. The Nature of the Chemical Bond, Cornell University Press 1940, Chap. X.

9. Weast, R. C. Handbook of Chemistry and Physics, 48th Ed., The Chemical Rubber Publishing Co., Cleveland, Ohio 1967-1968, E-58.

10. Hald, A. Statistical Theory with Engineering Applications, Wiley, New York 1965, pp. $551-557$.

Received April 19, 1969.

Acta Chem. Scand. 23 (1969) No. 9 